\title{
Modificação química superficial de fibras de bucha vegetal visando à compatibilização e aplicação como reforço em matriz cimentícia
}

\author{
Surface chemical modification of sponge gourd fiber \\ aiming at compatibility and application as reinforcement \\ in cementitious matrix
}

\section{José Diego Gasques Tolentino de Souza Leila Aparecida de Castro Motta Daniel Pasquini Júlia Graciele Vieira Cristiane Pires}

\section{Resumo}

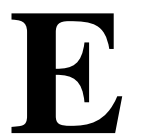

ste trabalho apresenta estudo da esterificação de fibras de bucha vegetal (Luffa cilyndrica) para verificar o desempenho delas na matriz cimentícia. As reações de esterificação foram realizadas com os agentes modificadores cloreto de octanoíla, cloreto de lauroíla e cloreto de estearoil, usando tolueno como solvente e piridina como catalisador. As fibras foram caracterizadas por ensaios de tração direta, microscopia eletrônica de varredura (MEV) e absorção de umidade. Foram produzidos compósitos com as fibras sem tratamento e tratadas com cloreto de octanoíla, tendo estas apresentado os melhores resultados, com redução de $65 \%$ na absorção de umidade, $40 \%$ na resistência à tração, e aumento de $67 \%$ no módulo de elasticidade. Os resultados do ensaio de flexão dos compósitos indicaram que a tenacidade da ordem de 0,70 $\mathrm{kJ} / \mathrm{m}^{2}$ não se alterou significativamente e que houve aumento de cerca de $20 \%$ no módulo de ruptura (6,64 MPa) e no módulo de elasticidade (15,10 GPa) dos compósitos reforçados com as fibras modificadas em relação aos reforçados com as fibras sem tratamento. As melhores propriedades mecânicas do compósito com as fibras tratadas indicam que o tratamento proposto pode ter melhorado o

José Diego Gasques Tolentino de Souza Universidade Federal de Uberlândia Mirassol - SP - Brasil

Leila Aparecida de Castro Motta Universidade Federal de Uberlândia Uberlândia - MG - Brasil

Daniel Pasquini Universidade Federal de Uberlândia Uberlândia - MG - Brasil

Júlia Graciele Vieira Universidade Federal de Uberlândia Ituiutaba - MG - Brasil

Cristiane Pires Universidade Federal de Uberlândia Uberlândia - MG - Brasil

Recebido em 02/11/15 Aceito em 07/07/16 desempenho das fibras vegetais como reforço da matriz cimentícia.

Palavras-chaves: Fibras vegetais. Bucha vegetal. Esterificação. Compósitos. Absorção de umidade. Propriedades mecânicas.

\section{Abstract}

This paper discusses the esterification of sponge gourd fibres (Luffa cilyndrica) to reinforce the cementitious matrix. The esterification reactions were performed with the modifying agents octanoyl chloride, lauroyl chloride and stearoyl chloride, using toluene as solvent and pyridine as catalyst. The fibres were characterised through tensile tests, scanning electron microscopy (SEM) and moisture absorption. Composites were produced with untreated and treated fibres with octanoyl chloride, which yielded the best results, with a $65 \%$ reduction in moisture absorption, $40 \%$ in tensile strength and $67 \%$ increase in the elasticity modulus. The results of the composites bending test indicated that a toughness of $0.70 \mathrm{~kJ} / \mathrm{m}^{2}$ did not change significantly and there was an increase of about $20 \%$ in the rupture modulus (6.64 MPa) and the elasticity modulus (15.10 GPa) of the composites reinforced with treated fibres compared with those reinforced with untreated fibres. The best mechanical properties of the composites with treated fibres indicate that the proposed treatment may have improved the performance of sponge gourd fibres as a reinforcement of the cementitious matrix.

Keywords: Vegetable fibers. Sponge gourd fiber. Esterification. Composites. Moisture absorption. Mechanical properties. 


\section{Introdução}

As fibras vegetais apresentam uma série de vantagens, o que justifica seu uso como reforço em matrizes cimentícias e compósitos. Entre as diversas vantagens podem-se destacar (MARINELLI et al., 2008; FARUK et al., 2012; FIORE et al., 2015):

(a) fibras vegetais são materiais renováveis, e sua disponibilidade pode ser considerada ilimitada;

(b) devido à enorme diversidade de plantas lenhosas e fibrosas encontradas na biodiversidade, existe grande potencial para a descoberta de fibras naturais com propriedades desejáveis;

(c) compósitos reforçados com fibras naturais, que também utilizam matrizes biodegradáveis, são considerados os materiais menos agressivos ao meio ambiente;

(d) possuem baixa densidade e alta ductilidade quando comparadas com materiais similares nesse campo de aplicação;

(e) apresentam baixo custo em relação aos reforços atualmente empregados; e

(f) não são prejudiciais à saúde.

No entanto, as fibras vegetais apresentam desvantagens, entre as quais se podem destacar: sua durabilidade na matriz cimentícia e a manutenção da integridade da interface fibra/matriz (AGOPYAN et al., 2005); alta alcalinidade da matriz cimentícia, que enfraquece as fibras de celulose, induzindo a sua mineralização (WEI; MEYER, 2015); e, consequentemente, diminuição da tenacidade do compósito em longo prazo. Além disso, em condições severas de intemperismo, o compósito absorve água, o que resulta em mudanças de volume da matriz de cimento e das fibras. Tem-se observado, em consequência desses ciclos de absorção de água, perda de aderência entre fibra e matriz de cimento, resultando em desarticulação dos elementos de reforço e deterioração das propriedades mecânicas dos compósitos (FERREIRA et al., 2012).

Para melhorar a durabilidade das fibras vegetais utilizadas como reforço em compósitos à base de cimento, várias abordagens têm sido estudadas, como impregnação com agentes bloqueadores e repelentes de água, agentes de vedação nos poros da matriz, redução da alcalinidade da matriz e combinações de impregnação e modificação da matriz (SILVA, 2009; MOTTA, 2006; MOTTA; JOHN; AGOPYAN, 2010; TONOLI et al., 2013; WEI; MEYER, 2015).
O mercado do fibrocimento é o que oferece o maior desafio quanto à busca de fibras para uso como elemento de reforço e de processamento, em substituição ao amianto. Matrizes à base de cimento reforçadas com fibras celulósicas têm-se mostrado promissoras quando o objetivo é obter um material com baixo custo, capaz de economizar energia e, principalmente, preservar o meio ambiente. As matrizes frágeis, como é o caso das matrizes à base de cimento, reforçadas com fibras têm ganhado espaço como material de construção em âmbito mundial, principalmente pela possibilidade de produção de elementos delgados em variadas formas e com elevada tenacidade (TOLÊDO FILHO et al., 2000; HOSSEINPOURPIA et al., 2012).

\section{Compatibilização da celulose}

Tendo o conhecimento das grandes diferenças de propriedades superficiais entre as fibras celulósicas e a matriz cimentícia, já que as primeiras são altamente polares e hidrofílicas, a modificação química da superfície das fibras celulósicas (Figura 1) emerge como alternativa para melhorar a compatibilidade celulose-matriz e a aderência interfacial, tornando a fibra hidrofóbica (TONOLI et al., 2013).

Sem tal tratamento as fibras naturais incorporadas na matriz cimentícia geram instabilidade, e qualquer estresse aplicado às interfaces da mistura fibras-matriz não é eficientemente transferido da matriz para as fibras e suas propriedades permanecem subexploradas (TONOLI et al., 2013; WEI; MEYER, 2015).

\section{Fibra de bucha vegetal}

No Brasil somente no ano de 2006 foram produzidas 1.198 toneladas de bucha vegetal (IBGE, 2016). Atualmente, o cultivo compreende as regiões Norte e Nordeste, e também São Paulo, Minas Gerais e Mato Grosso, sendo Minas Gerais o principal produtor de bucha do país, ao contribuir com $50 \%$ da safra. Embora não haja dados oficiais sobre plantações comerciais no país, a cidade de Bonfim, MG, é considerada a capital da bucha natural, com produção anual de 100 mil dúzias (CARVALHO, 2007).

A bucha vegetal é pertencente à família das Curcubitaceae, planta proveniente de regiões da Ásia. As curcubitáceas estão entre as maiores e mais diversas famílias de plantas, sendo cultivadas em todo o mundo, por suportar uma variedade de condições ambientais (BISOGNIN, 2002). 
A planta, conhecida como trepadeira herbácea com caule anguloso, atinge comprimento de aproximadamente $10 \mathrm{~m}$. Já o fruto possui formato cilíndrico, com comprimento de $15 \mathrm{~cm}$ a $100 \mathrm{~cm}$ e diâmetro de $8 \mathrm{~cm}$ a $10 \mathrm{~cm}$ (ANNUNCIADO, 2005; TANOBE et al., 2002; TANOBE, 2003). O fruto da planta da bucha vegetal (Figura 2) tem um sistema vascular fibroso, no qual está disposta uma matriz multidirecional, que forma uma manta natural, com cor amarela quando maduro e castanho-escuro quando seco (D'ALMEIDA et al., 2005; SHEN et al., 2014).

$\mathrm{Na}$ indústria os frutos da bucha vegetal são utilizados para fabricação de palmilhas, chinelos, solados, peneiras, correias e filtros de óleos para automóveis, esponjas de banho e chapéus (ANNUNCIADO, 2005; TANOBE, 2003). Neste trabalho são estudados como reforço de compósitos em matriz de cimento.

\section{Programa experimental}

\section{Obtenção e preparação das fibras de bucha vegetal}

Foram utilizadas buchas vegetais de metro inteiras (Luffa cilyndrica), in natura, sem cascas e sementes, adquiridas do mercado em Uberlândia, MG, Brasil.

O processo de preparo consistiu em abertura da bucha vegetal, retirada das sementes remanescentes e corte com comprimento e largura de aproximadamente $20,5 \mathrm{~cm}$.

Posteriormente, as fibras foram compactadas na condição saturada, com aplicação de carga de 400 $\mathrm{kN}$ em uma prensa hidráulica por $24 \mathrm{~h}$. Com as fibras compactadas, obteve-se uma espécie de manta, aproveitando-se, assim, a estrutura reticulada do material (Figura 3). Após a prensagem, as mantas foram secas em estufa por $24 \mathrm{~h}$ à temperatura de $60^{\circ} \mathrm{C}$, resultando em uma espessura média de $0,75 \mathrm{~mm}$.

\section{Determinação da composição química das fibras}

O teor de lignina Klason foi determinado de acordo com a norma TAPPI T222 om-98 (TECHNICAL..., 1999). Já os teores de celulose e hemicelulose foram determinados segundo a norma TAPPI T235 cm-00 (TECHNICAL..., 2000).

\section{Figura 1 - Esquema da esterificação da celulose}

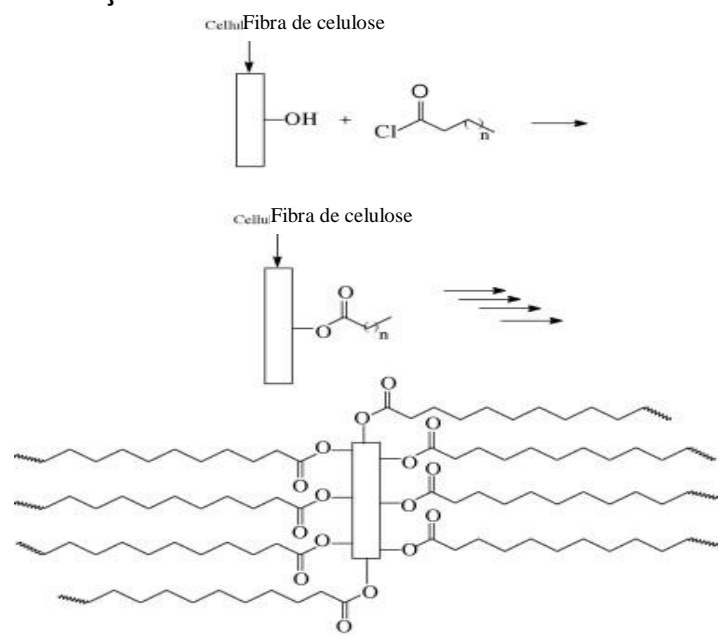

Fonte: adaptado de Gatenholm e Felix (1993).

Figura 2 - Fruto da bucha vegetal

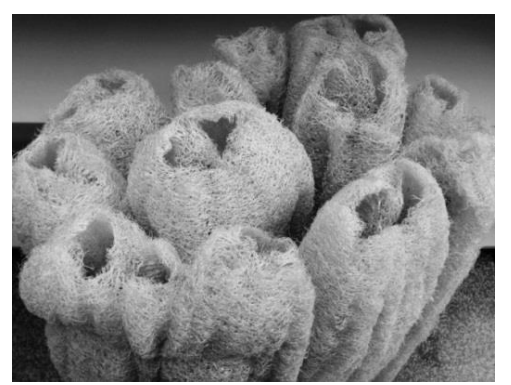


Figura 3 - Bucha vegetal recortada natural (à esquerda) e compactada (à direita)

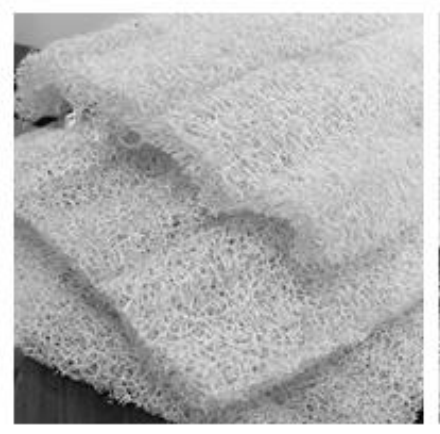

\section{Lignina Klason}

Primeiramente $1,00 \mathrm{~g}$ de bucha vegetal, livre de extrativos, foi transferido para um balão. Foram adicionados $30 \mathrm{~mL}$ de ácido sulfúrico (72\%), lentamente e sob agitação. A amostra foi então mantida durante $2 \mathrm{~h}$ em banho à temperatura ambiente $\left(25^{\circ} \mathrm{C}\right)$, sob agitação. Ao conteúdo do balão, foram então adicionados $560 \mathrm{~mL}$ de água destilada. O sistema foi colocado sob refluxo a uma temperatura de $100{ }^{\circ} \mathrm{C}$, para que não ocorresse perda de água por evaporação e, consequentemente, alteração na concentração da solução de ácido. Após 4 h, o sistema foi deixado em repouso para a sedimentação do material insolúvel. Esse material foi filtrado em funil de placa porosa, previamente tarado, e lavado com $500 \mathrm{~mL}$ de água destilada quente. Em seguida foi seco em estufa a $105^{\circ} \mathrm{C}$ por $12 \mathrm{~h}$ e pesado para quantificação do resíduo insolúvel (lignina Klason).

\section{Obtenção da holocelulose}

Primeiramente 5,0 g da amostra foram colocados em um balão. Adicionaram-se $100 \mathrm{~mL}$ de água destilada. O balão foi colocado em banho-maria a $75^{\circ} \mathrm{C}$, e adicionaram-se $2,0 \mathrm{~mL}$ de ácido acético e $3,0 \mathrm{~g}$ de clorito de sódio, nesta ordem, tampando o balão para não ocorrer a perda do gás produzido na reação. Após $1 \mathrm{~h}$ adicionaram-se novamente $2,0 \mathrm{~mL}$ de ácido acético e 3,0 g de clorito de sódio. Esse processo foi repetido por mais duas vezes. A mistura foi então resfriada a $10^{\circ} \mathrm{C}$, filtrada em funil de placa porosa, previamente tarado, e lavada com água destilada a $5{ }^{\circ} \mathrm{C}$ até que o resíduo fibroso apresentasse coloração esbranquiçada. $\mathrm{O}$ funil com o resíduo fibroso foi então seco em estufa a $105^{\circ} \mathrm{C}$ por $6 \mathrm{~h}$, resfriado em dessecador e pesado para se quantificar o rendimento da holocelulose.

\section{Determinação da celulose}

Transferiram-se 3,00 g de holocelulose para um Erlenmeyer de $250 \mathrm{~mL}$. Adicionaram-se $100 \mathrm{~mL}$ de solução de $\mathrm{KOH}(5 \%)$ e fez-se uma atmosfera inerte pela passagem de gás nitrogênio durante os 5 min iniciais da extração para evitar a oxidação da

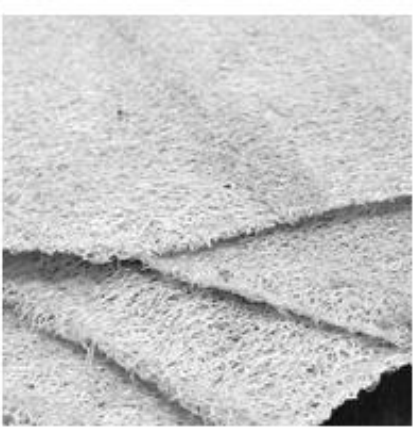

celulose. O Erlenmeyer foi vedado e mantido em agitação constante por $2 \mathrm{~h}$. A mistura foi então filtrada em funil de placa porosa, lavada com 50 $\mathrm{mL}$ de solução de $\mathrm{KOH}(5 \%)$ e, em seguida, com $100 \mathrm{~mL}$ de água destilada. O filtrado foi então recolhido em um Erlenmeyer de $1 \mathrm{~L}$ e precipitado com uma solução de partes iguais de ácido acético e etanol (completando-se o volume do Erlenmeyer), obtendo-se assim a hemicelulose A.

Para a obtenção da hemicelulose $\mathrm{B}$, o resíduo fibroso retido no funil foi transferido novamente para o Erlenmeyer de $250 \mathrm{~mL}$. O mesmo procedimento para a obtenção da hemicelulose A foi repetido utilizando-se solução de $\mathrm{KOH}$ (24\%). Para lavagem do resíduo fibroso retido no funil utilizaram-se $25 \mathrm{~mL}$ de solução de $\mathrm{KOH}$ (24\%), 50 $\mathrm{mL}$ de água destilada, $25 \mathrm{~mL}$ de ácido acético $(10 \%)$ e $100 \mathrm{~mL}$ de água destilada. O filtrado recolhido em Erlenmeyer de $1 \mathrm{~L}$ foi precipitado com uma solução de partes iguais de ácido acético e etanol (completando-se o volume do Erlenmeyer), obtendo-se assim a hemicelulose B.

Após a extração dos componentes solúveis em soluções aquosas de hidróxido de potássio, o resíduo fibroso foi lavado com água destilada até que o filtrado apresentasse $\mathrm{pH}$ neutro. $\mathrm{O}$ resíduo foi então lavado com $50 \mathrm{~mL}$ de acetona, seco a $105^{\circ} \mathrm{C}$ e pesado. Esse resíduo é denominado celulose.

\section{Modificação química das fibras}

Para a modificação superficial das fibras de bucha vegetal foram adotadas as recomendações de Pasquini et al. (2008). Utilizou-se um sistema de refluxo na temperatura de ebulição do solvente por $1 \mathrm{~h}$ (Figura 4a), em que ocorreram as reações com os diferentes agentes modificadores, cloreto de octanoíla, cloreto de lauroíla e cloreto de estearoil, todos em solução de tolueno (solvente) e piridina (catalisador).

Foi utilizado $0,50 \mathrm{~mL}$ de cada agente modificador, em solução de $50,00 \mathrm{~mL}$ de tolueno e $10,0 \mathrm{~mL}$ de piridina para cada $1,0 \mathrm{~g}$ de fibra. Repetiu-se o experimento variando-se as quantidades dos 
agentes modificadores, com $0,75 \mathrm{~mL}$ e $1,0 \mathrm{~mL}$, obedecendo-se às proporções.

Após as modificações as fibras foram lavadas em Soxhlet com acetona durante $20 \mathrm{~h}$ para eliminação dos resíduos de reagentes (Figura $4 \mathrm{~b}$ ). $\mathrm{O}$ aspecto e a integridade da manta não foram alterados com o tratamento.

\section{Microscopia eletrônica de varredura (MEV)}

Amostras de fibras modificadas e sem tratamento foram analisadas por microscopia eletrônica de varredura (MEV) com o objetivo de avaliar as modificações morfológicas pelo tratamento químico superficial. O recobrimento das amostras foi feito com ouro, e o microscópio eletrônico de varredura usado foi da marca Carl Zeiss, modelo EVO MA10 (Alemanha), feixes de $5 \mathrm{kV}$ e detector de elétrons secundários.

\section{Ensaio de absorção de umidade}

A efetividade da reação de esterificação sobre a superfície da fibra vegetal pode ser verificada por medidas de espectroscopia de infravermelho com Transformada de Fourier (FTIR), medidas de ângulo de contato, absorção de água, entre outros tipos de caracterizações. Neste trabalho optou-se por realizar o ensaio de absorção de umidade das fibras de bucha vegetal sem tratamento e modificadas para determinar o nível de hidrofobicidade das fibras. Para o procedimento utilizou-se um dessecador com solução saturada de nitrato de sódio para criar uma atmosfera com umidade de 95\%. A medição e o controle de temperatura e umidade foram realizados com um termo-higrômetro, marca Minipa, modelo MT-241. Todas as fibras foram secas em estufa à temperatura de $100{ }^{\circ} \mathrm{C}$ por $24 \mathrm{~h}$. As fibras foram colocadas no dessecador e realizadas medidas de massa nos intervalos de 1, 2, 24, 48, 72 e $120 \mathrm{~h}$.

\section{Ensaio de tração direta das fibras}

Para a determinação da resistência à tração, deformação na ruptura e módulo de elasticidade das fibras seguiu-se o método adotado por Motta, John e Agopyan (2010).

A deformação das fibras foi determinada pela relação entre $\mathrm{o}$ alongamento medido e o comprimento livre inicial da fibra. $\mathrm{O}$ alongamento da fibra foi medido pelo afastamento das garras. Devido às reduzidas dimensões dos corpos de prova não foi possível a utilização de instrumentos de medida de deformação ou deslocamento, como extensômetros elétricos ou LVDT. Utilizando um LVDT INSTRON verificou-se inicialmente que o afastamento da garra e o real deslocamento não apresentavam diferença significativa. Os resultados obtidos apresentaram boa correlação, com erro desprezível. Todas as medidas para evitar eventuais escorregamentos e acomodações durante o ensaio foram sempre tomadas.

O ensaio de tração foi realizado na fibra, e não em feixes de fibras, como mostrado nas Figuras 5 e 6. Para isso, estas foram recortadas da bucha em comprimento mínimo de $6 \mathrm{~mm}$.

Figura 4 - Modificação química das fibras de bucha vegetal

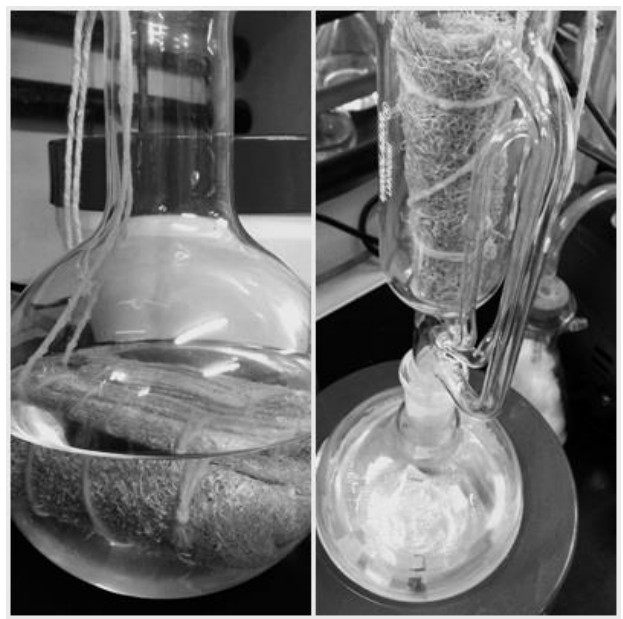

(a) Esterificação $\quad$ (b) Lavagem 
Figura 5 - Imagens dos corpos de prova para ensaio de tração das fibras

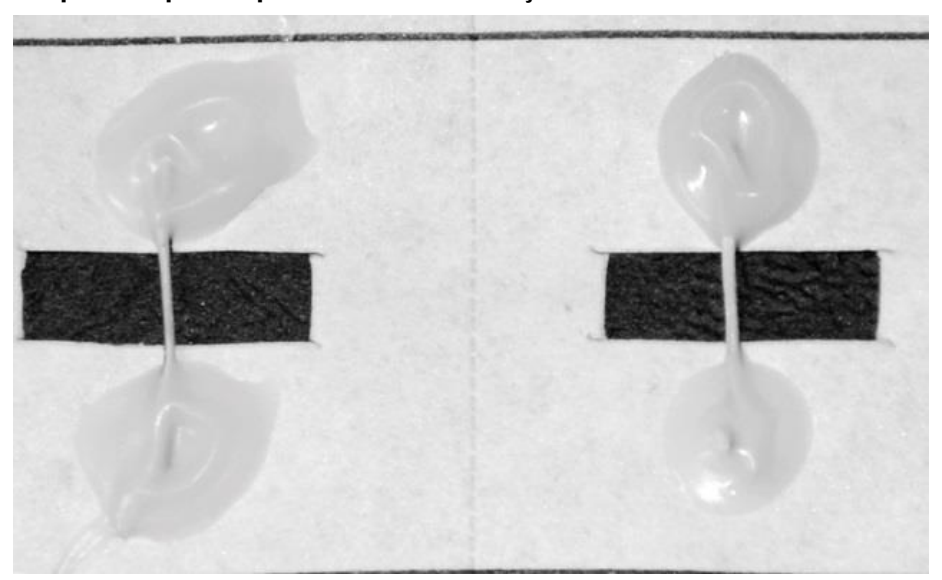

As fibras foram posicionadas e coladas em base de papel. Em seguida, para se determinarem os diâmetros e os comprimentos das fibras utilizou-se análise de imagem com o software Image Tool (Zeiss, Alemanha), em que cada corpo de prova foi fotografado em microscópio óptico da marca Olympus, modelo CX40. As imagens foram obtidas com câmera de alta resolução, marca Zeiss, modelo ICc5. Por análise de imagem, determinaram-se os diâmetros e comprimentos das fibras (corpos de prova), sendo a seção transversal destas calculada considerando sua seção circular aplicando-se fator de correção como descrito em Motta, John e Agopyan (2010).

Posteriormente, quando fixadas nas garras da máquina de ensaio, cortaram-se as laterais da base de papel, ensaiando-se somente a fibra à tração, conforme pode ser visto na Figura 6.

A distância entre as garras da máquina coincide com o comprimento útil do corpo de prova (fibra). A máquina universal de ensaio usada foi da marca Instron, modelo 5982 (EUA), e célula de carga de 1 $\mathrm{kN}$. A faixa de carga de ruptura obtida para as fibras estava dentro do limite de precisão da célula de carga utilizada. A velocidade adotada nos ensaios foi de $2 \mathrm{~mm} / \mathrm{min}$. Para cada condição foram ensaiadas 20 fibras com comprimento útil de ensaio igual a $3 \mathrm{~mm}$.

\section{Preparação dos compósitos \\ Mistura e moldagem}

O processo de mistura e preparação dos compósitos foi uma simulação do processo Hatschek para moldagem de fibrocimento, com mistura das fibras celulósicas em calda de cimento e água, com posterior remoção do excesso de água por vácuo, e, por fim, prensagem com pressão de aproximadamente $\quad 3,2 \quad \mathrm{MPa} \quad$ (SAVASTANO JUNIOR, 2000).
A formulação básica usada para a mistura continha cimento, água e polpa celulósica de eucalipto. O cimento utilizado foi o CPIII 40 RS da marca Cauê, produzido pela Intercement (Brasil), e a água foi da rede pública. A polpa celulósica empregada foi a de eucalipto branqueada, utilizada unicamente para permitir a moldagem por sucção, sendo sempre igual a $2 \%$ em volume, o que corresponde ao volume mínimo necessário para filtrar os finos da mistura durante a sucção do excesso de água. A concentração inicial de sólidos na mistura foi de $45 \%$ em massa. O teor de fibras de bucha foi de $3 \%$ em volume, usada na forma de manta (tecido com a estrutura natural da bucha) como ilustrado na Figura 3. A manta foi posicionada na porção média do compósito, como pode ser visto na Figura 8. Não houve segregação, e a matriz homogênea recobre a manta nas duas faces.

Para a mistura dos constituintes, inicialmente se colocou toda a água no misturador, seguida da polpa de celulose, dispersando-se esta por $5 \mathrm{~min}$. Adicionou-se então o cimento e misturou-se por mais 5 min. A Figura 7 traz um esquema mostrando a sequência de preparação dos compósitos.

A cura das placas moldadas se deu em câmara úmida com umidade relativa acima de $95 \%$ e temperatura igual a $23 \pm 2{ }^{\circ} \mathrm{C}$ durante os primeiros 28 dias de idade. As placas moldadas possuem dimensões de 200x200 mm de largura e $8 \mathrm{~mm}$ de espessura média.

Os corpos de prova (Figura 8) para o ensaio de flexão foram obtidos das placas. Após os primeiros 21 dias de idade, ainda durante o período de cura, os corpos de prova foram cortados nas dimensões aproximadas de $180 \mathrm{~mm}$ de comprimento por 40 $\mathrm{mm}$ de largura utilizando serra de disco.

\section{Ensaios de flexão dos compósitos}

Os corpos de prova extraídos das placas foram submetidos a ensaios de flexão aos 28 dias de idade. 
Com o ensaio de flexão foram determinados módulo de ruptura (MOR), módulo de elasticidade e tenacidade dos compósitos. Foram ensaiados 4 corpos de prova para cada formulação, na idade de 28 dias, na condição saturada.

Os parâmetros adotados para a realização dos ensaios de flexão foram baseados em Motta (2006).
Utilizou-se uma máquina universal da marca Instron, modelo 5982, com célula de carga com capacidade de 5,0 kN. Aplicou-se o método de flexão em 4 pontos (Figura 9) e velocidade automática do deslocamento igual a $2,0 \mathrm{~mm} / \mathrm{min}$. A flecha no centro do vão foi medida com um LVDT da marca Instron, com curso máximo de $5 \mathrm{~mm}$.

Figura 6 - Ensaio de tração direta das fibras

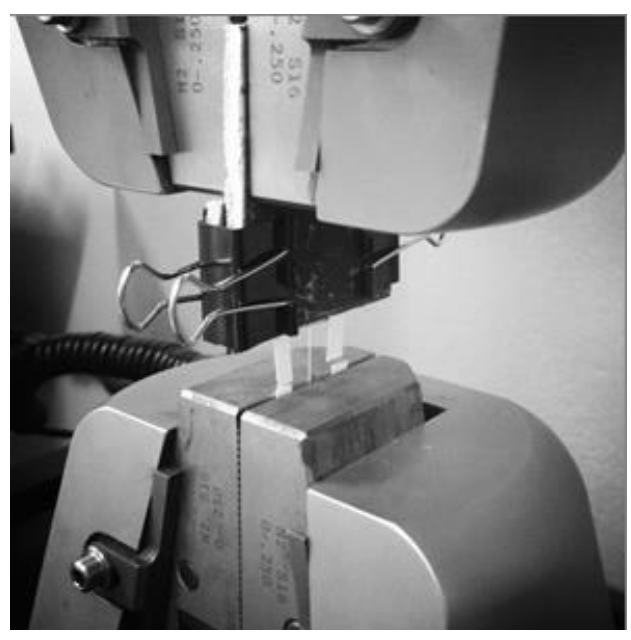

Figura 7 - Esquema ilustrando a mistura e a moldagem dos compósitos

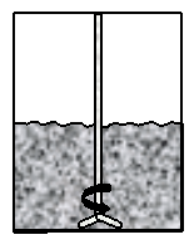

Mistura

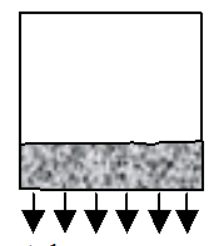

Adensamento por sucção

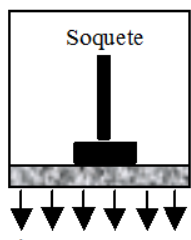

Adensamento por sucção e manual

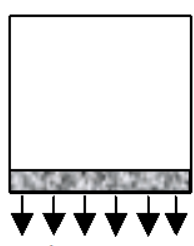

Adensamento

por sucção

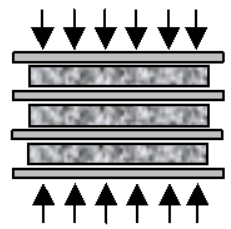

Prensagem

Fonte: Motta (2006).

Figura 8 - Corpos de prova após cura de 28 dias e corte

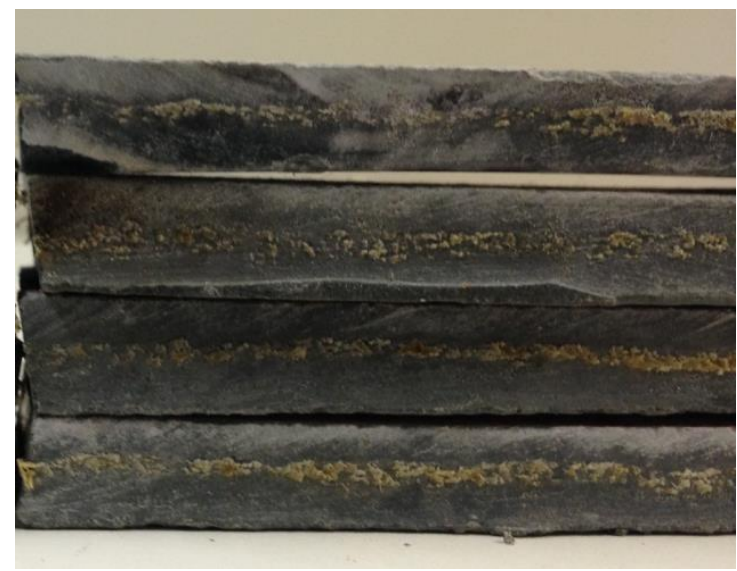


Figura 9 - Ensaio de flexão em quatro pontos

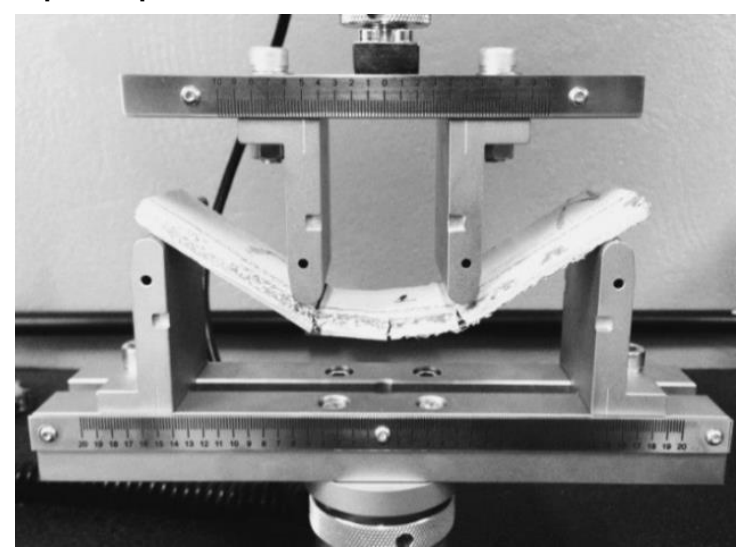

O módulo de ruptura (MOR) foi determinado com a expressão da Eq. 1, e o módulo de elasticidade calculado pela Eq. 2 .

$$
\begin{aligned}
& \text { MOR }=\frac{P_{\text {máx }} L}{b e^{2}} \\
& E=\frac{23 L^{3}}{1296 I}\left(\frac{P}{\delta}\right)
\end{aligned}
$$

Onde:

(a) $P_{\text {máx }}=$ força máxima durante o ensaio $(\mathrm{N})$;

(b) $L=$ distância entre apoios inferiores. A distância entre os pontos de aplicação da carga e os apoios inferiores é igual a $L$ dividido por $3(\mathrm{~mm})$;

(c) $b=$ largura do corpo de prova $(\mathrm{mm})$;

(d) $e=$ espessura do corpo de prova $(\mathrm{mm})$;

(e) $E=$ módulo de elasticidade (MPa);

(f) $I=$ momento de inércia da seção transversal $\rightarrow$ $\frac{b e^{3}}{12}\left(\mathrm{~mm}^{4}\right) ; \mathrm{e}$

(g) $P / \delta=$ coeficiente angular da reta obtida da curva $\mathrm{P}$ x $\delta$ (força $\mathrm{x}$ flecha) no trecho elástico $(\mathrm{N} / \mathrm{mm})$.

A tenacidade do compósito foi determinada pela integração da área sob a curva força x flecha resultante do ensaio de flexão, até o ponto de flecha máxima (final do ensaio), dividida pela área da seção transversal dos corpos de prova, expressa em $\mathrm{kJ} / \mathrm{m}^{2}$.

Para todos os resultados obtidos para as propriedades mecânicas das fibras e compósitos foi aplicado o critério de Chauvenet para exclusão de dados espúrios.

\section{Resultados e discussão}

\section{Composição química das fibras}

Os componentes químicos das fibras de bucha vegetal utilizadas neste estudo estão discriminados na Tabela 1.

\section{Microscopia eletrônica de varredura (MEV)}

Observando-se as micrografias, apenas se pode afirmar que a modificação química foi modesta a ponto de não ocorrer mudanças significativas na estrutura das fibras naturais (Figuras 11 e 12). No entanto, o processo de esterificação de fibras lignocelulósicas é muito bem conhecido na literatura e já foi amplamente comprovado (PASQUINI et al. 2006, 2008; FREIRE et al., 2006a, 2006b). Apenas uma pequena modificação na superfície foi observada pela geração de irregularidades, conforme exibido nas imagens de MEV (Figuras 13 e 14).

\section{Ensaio de absorção de umidade}

Os resultados mostraram que houve redução na absorção de umidade das fibras modificadas, o que se deve ao fato de a modificação química ter hidrofobizado as fibras. Esse comportamento não pode ser atribuído à lavagem das fibras por tolueno, pois, se fosse este o caso, o tolueno agiria removendo os compostos apolares presentes nas fibras, tais como gorduras, ceras e outros compostos orgânicos apolares, e como resultado deixaria as fibras mais polares e, consequentemente, estas absorveriam mais umidade.

Observou-se que as fibras modificadas com cloreto de lauroíla na concentração de $1 \mathrm{~mL} / \mathrm{g}$ e de 0,75 $\mathrm{mL} / \mathrm{g}$ apresentaram os menores valores de absorção, com redução de $70 \%$ e $67 \%$ respectivamente em relação à fibra sem tratamento. As fibras esterificadas com cloreto de octanoíla a $0,75 \mathrm{~mL} / \mathrm{g}$ também apresentaram menor absorção de umidade, com redução de $64 \%$, como mostra a Figura 14. A fibra com menor absorção poderá ter reduzida a migração de produtos de hidratação para seu interior. 
Tabela 1 - Composição química das fibras de bucha vegetal

\begin{tabular}{c|c}
\hline Componentes & Porcentagem \\
\hline Celulose & $70,8( \pm 0,5)$ \\
Lignina & $14,7( \pm 0,7)$ \\
Hemiceluloses A e B & $17,2( \pm 0,8)$ \\
\hline
\end{tabular}

Figura 10 - Seção transversal da fibra natural

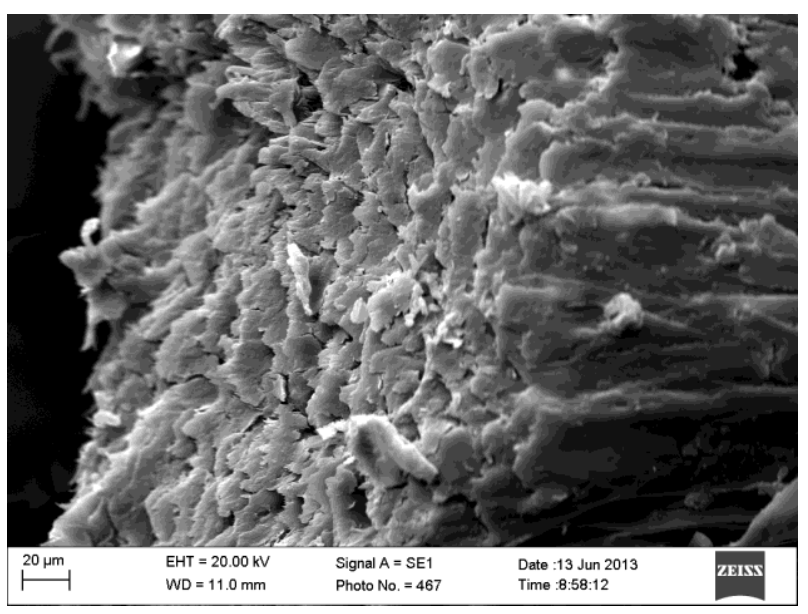

Figura 11 - Superfície da fibra in natura

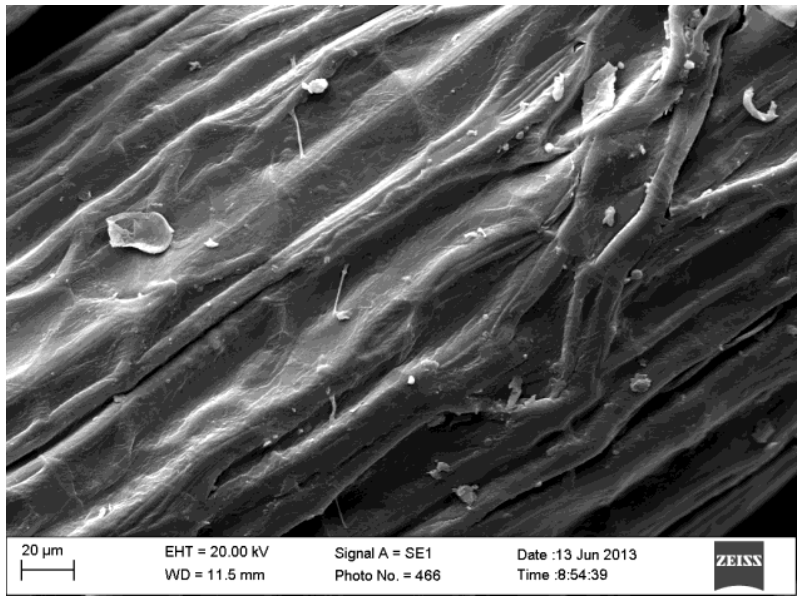

Figura 12 - Seção transversal da fibra modificada

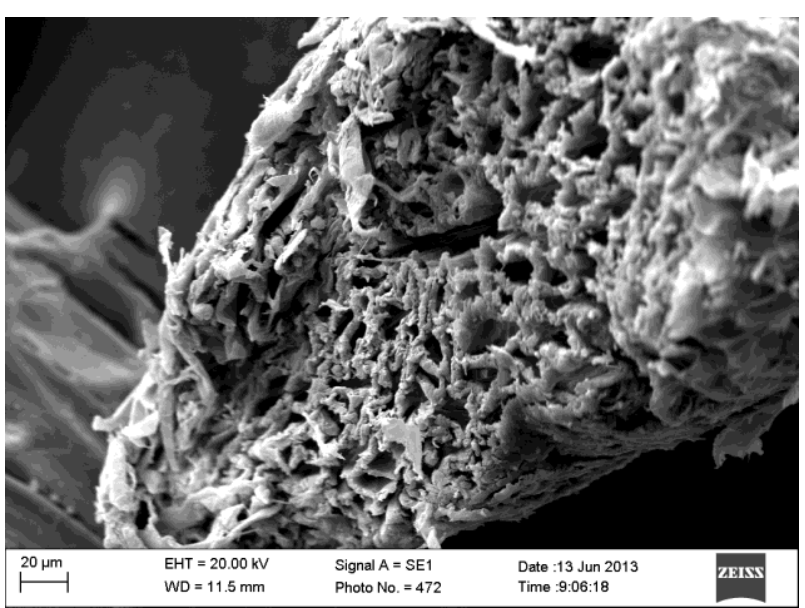


Figura 13 - Superfície da fibra modificada

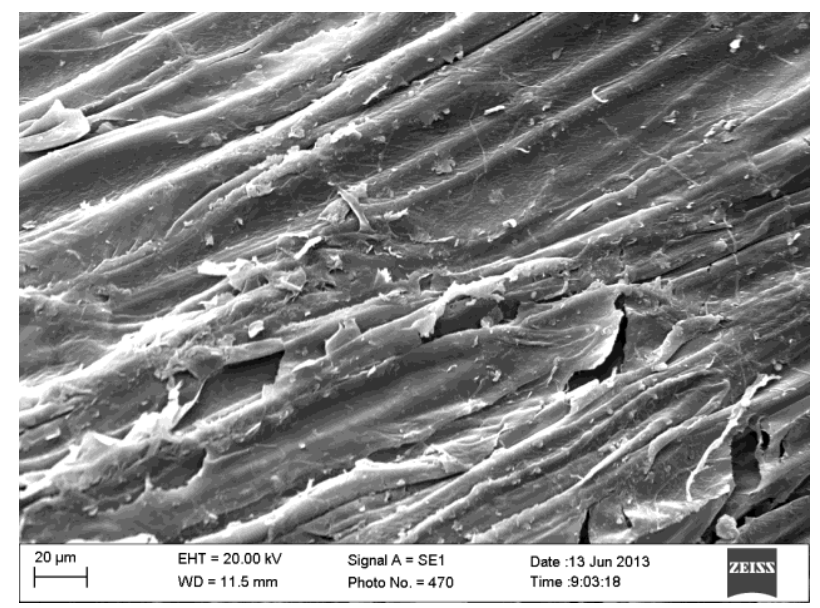

Figuras 14 - Isotermas de absorção de umidade das fibras

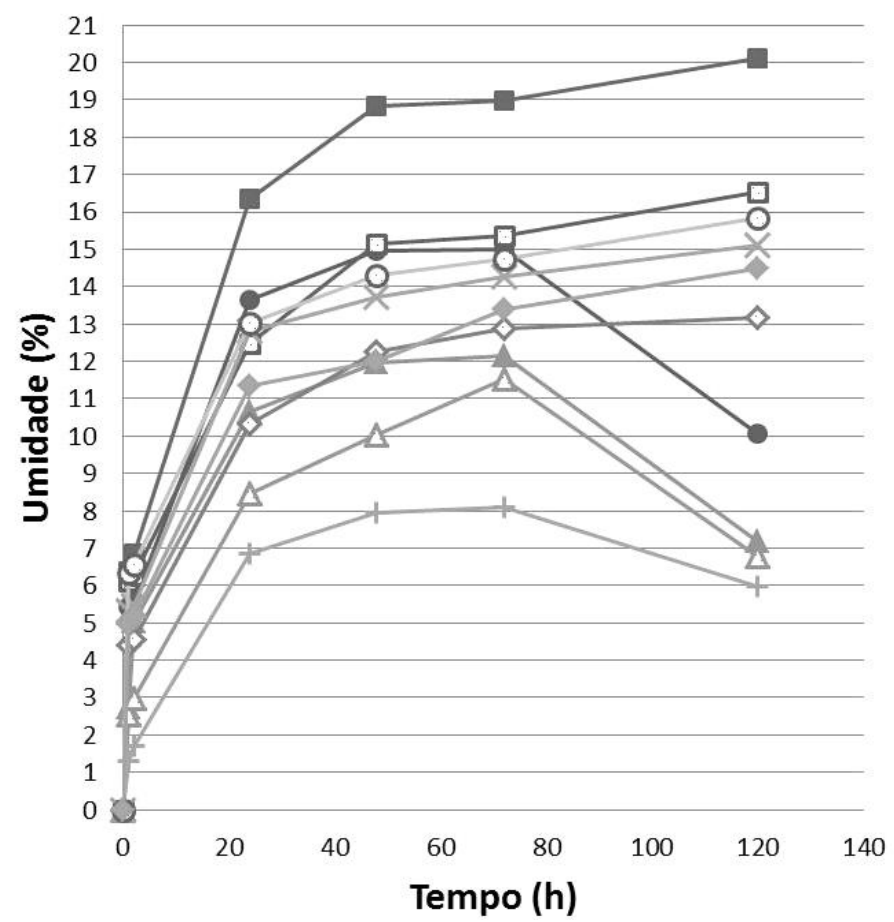

$$
\begin{aligned}
& - \text { - Fibra natural } \\
& \text { - Fibra modificada - Cloreto de } \\
& \text { Octanoíla 0,5mL/g } \\
& \text { - Fibra modificada - Cloreto de } \\
& \text { Octanoíla 0,75mL/g } \\
& -\square-\text { Fibra modificada - Cloreto de } \\
& \text { Octanoíla } 1 \mathrm{~mL} / \mathrm{g} \\
& \checkmark-\text { Fibra modificada - Cloreto de } \\
& \text { Lauroíla 0,5mL/g } \\
& \triangle \text { Fibra modificada - Cloreto de } \\
& \text { Lauroíla 0,75mL/g } \\
& \text { — Fibra modificada - Cloreto de } \\
& \text { Lauroíla } 1 \mathrm{~mL} / \mathrm{g} \\
& \leftarrow \text { Fibra modificada - Cloreto de } \\
& \text { Estearoil 0,5mL/g }
\end{aligned}
$$

A menor absorção de umidade das fibras reduz sua variação dimensional, o que pode melhorar a interação entre matriz e fibras, como discutido por Ferreira et al. (2012), evitando o descolamento das fibras da matriz em ciclos naturais de molhagem e secagem.

\section{Ensaio de tração direta das fibras}

As propriedades mecânicas das fibras de bucha vegetal sem tratamento se assemelham às propriedades de outras fibras estudadas para ser utilizadas em compósitos cimentícios, como as fibras de coco (MOTTA, 2006).
Notou-se que houve redução das propriedades mecânicas com o tratamento químico, em que o cloreto de lauroíla foi o mais agressivo, com redução de $80 \%$ na resistência à tração das fibras modificadas. As fibras modificadas com cloreto de octanoíla e estearoil apresentaram resistência à tração, em média, 50\% menor, comparado à fibra sem tratamento. O módulo de elasticidade das fibras tratadas também sofreu redução, exceto para o tratamento com cloreto de octanoíla, em que, para a modificação com $0,5 \mathrm{~mL} / \mathrm{g}$, não houve alteração, e com $0,75 \mathrm{~mL} / \mathrm{g}$ o módulo de elasticidade teve aumento de $67 \%$.

Para as fibras modificadas com o cloreto de octanoíla na concentração de $1 \mathrm{~mL} / \mathrm{g}$ não foi 
possível executar o ensaio de tração, pois as fibras estavam bastante deterioradas. A Tabela 2 contém os valores médios com respectivos desvios padrão obtidos nos ensaios dos 20 corpos de prova para cada condição.

\section{Ensaio de flexão dos compósitos}

Utilizou-se como referência o compósito com cimento Portland e fibras como recebidas (in natura), pois o objetivo do trabalho foi comparar o desempenho das fibras tratadas no compósito para avaliar a eficiência do tratamento. A melhora das propriedades mecânicas com a presença das fibras em relação à matriz cimentícia sem fibra é bem conhecida na literatura (TOLÊDO FILHO et al., 2003; AGOPYAN et al., 2005; FERREIRA et al., 2012) e não foi objeto de análise deste trabalho.

A melhor condição de modificação química superficial para o tratamento das fibras foi escolhida considerando-se os resultados obtidos no ensaio de tração direta das fibras, pois as propriedades mecânicas (resistência à tração e módulo de elasticidade) são fundamentais para a aplicação das fibras como reforço.

Além disso, utilizou-se também como parâmetro de escolha o ensaio de absorção de umidade, para melhorar a impermeabilização das fibras e, com isso, seu desempenho como reforço na matriz de cimento. Portanto, considerando a expressiva queda na absorção de umidade e a menor deterioração das propriedades mecânicas, utilizaram-se fibras de bucha vegetal tratadas com cloreto de octanoíla na concentração de $0,75 \mathrm{~mL} / \mathrm{g}$, para ser testadas nos compósitos.

Os resultados dos ensaios de flexão dos compósitos aos 28 dias de idade (Tabela 3) indicam que houve melhora nas propriedades mecânicas dos compósitos reforçados com as fibras modificadas em comparação aos reforçados com as fibras sem tratamento. A maior resistência à flexão e módulo de elasticidade dos compósitos com a fibra tratada, com aumento de aproximadamente $20 \%$ para ambas as propriedades mecânicas, pode ser devida à melhor interação das fibras modificadas com a matriz de cimento e também pelo melhor módulo de elasticidade da fibra tratada.

Foi possível perceber também que as fibras sem tratamento utilizadas como reforço nos compósitos apresentaram notável inchamento (recuperação da forma não compactada) quando da preparação dos compósitos, devido à absorção de água da mistura, o que não ocorreu com as fibras modificadas quimicamente, que se mantiveram compactadas. Essa observação está em conformidade com os resultados obtidos de menor absorção das fibras modificadas quimicamente.

Após a fissuração da matriz, a presença das fibras em forma de manta (estrutura reticulada) causa a redistribuição de tensões ao longo do material, o que permite o comportamento pós-pico observado nas Figuras 15 e 16.

Após a primeira fissura sob um dos pontos de carga, as tensões foram transferidas às fibras, que, resistentes e bem ancoradas na matriz pela forma de tecido com que foram usadas, permitiram o crescimento das tensões novamente (segundo pico) até a ocorrência da segunda fissura, sob o segundo ponto de carga.

Observou-se que nos compósitos com as fibras sem tratamento, mais resistentes, as fibras não são rompidas, e o crescimento das tensões após o segundo pico continua até a flecha atingir os limites do ensaio, aproximadamente $5 \mathrm{~mm}$. No entanto, para os compósitos com as fibras modificadas, algumas fibras são rompidas com o aumento da flecha, causando a redução das tensões ao longo do ensaio. Entretanto, esse comportamento não implicou redução da tenacidade, sendo a tenacidade dos dois compósitos estatisticamente similar, $0,69 \mathrm{~kJ} / \mathrm{m}^{2}$ e $0,72 \mathrm{~kJ} / \mathrm{m}^{2}$, com as fibras sem e com tratamento respectivamente.

\section{Conclusões}

O tratamento das fibras com cloreto de lauroíla foi o que mais reduziu a absorção de umidade, mas deteriorou muito as propriedades mecânicas das fibras tratadas. A modificação com cloreto de octanoíla $(0,75 \mathrm{~mL} / \mathrm{g})$ reduziu a absorção $(65 \%)$ e alterou menos a resistência à tração das fibras (redução de 40\%), com aumento de 67\% no módulo de elasticidade, sendo, portanto, considerada a melhor condição para o tratamento das fibras de bucha vegetal.

Aos 28 dias de idade, os compósitos reforçados com as fibras modificadas com cloreto de octanoíla $(0,75 \mathrm{~mL} / \mathrm{g})$, comparados aos reforçados com as fibras sem tratamento, apresentaram tenacidade similar $(0,72$ e 0,69 respectivamente $)$ e aumento de cerca de $20 \%$ no módulo de ruptura e no módulo de elasticidade, indicando melhor interação das fibras com a matriz, provavelmente devido à redução da absorção das fibras.

Estudos de durabilidade dos compósitos reforçados com as fibras modificadas com cloreto de octanoíla e demais condições de tratamento estão sendo realizados e serão publicados em breve. 
Tabela 2 - Propriedades obtidas nos ensaios de tração direta das fibras - Resultados médios com desvios padrão entre parênteses

\begin{tabular}{|c|c|c|c|}
\hline & $\begin{array}{l}\text { Resistência à } \\
\text { tração (MPa) }\end{array}$ & $\begin{array}{c}\text { Deformação } \\
\text { (mm/mm) }\end{array}$ & $\begin{array}{l}\text { Módulo de } \\
\text { elasticidade } \\
\text { (GPa) }\end{array}$ \\
\hline Fibra sem tratamento $(3 \mathrm{~mm})$ & $99,2(32,25)$ & $0,177(0,03)$ & $1,00(0,27)$ \\
\hline Fibra modificada - cloreto de octanoíla $0,5 \mathrm{~mL} / \mathrm{g}$ & $51,4(13.85)$ & $0,060(0,01)$ & $1,05(0,26)$ \\
\hline Fibra modificada - cloreto de octanoíla $0,75 \mathrm{~mL} / \mathrm{g}$ & $58,3(8,96)$ & $0,046(0,01)$ & $1,67(0,40)$ \\
\hline Fibra modificada - cloreto de octanoíla $1 \mathrm{~mL} / \mathrm{g}$ & n.d. & n.d. & n.d. \\
\hline Fibra modificada - cloreto de lauroíla $0,5 \mathrm{~mL} / \mathrm{g}$ & $18,5(6,62)$ & $0,059(0,01)$ & $0,48(0,06)$ \\
\hline Fibra modificada - cloreto de lauroíla $0,75 \mathrm{~mL} / \mathrm{g}$ & $17,4(5,12)$ & $0,077(0,03)$ & $0,36(0,10)$ \\
\hline Fibra modificada - cloreto de lauroíla $1 \mathrm{~mL} / \mathrm{g}$ & $20,2(3,67)$ & $0,066(0,01)$ & $0,41(0,08)$ \\
\hline Fibra modificada - cloreto de estearoil $0,5 \mathrm{~mL} / \mathrm{g}$ & $44,9(7,58)$ & $0,114(0,03)$ & $0,52(0,12)$ \\
\hline Fibra modificada - cloreto de estearoil $0,75 \mathrm{~mL} / \mathrm{g}$ & $30,3(5,39)$ & $0,083(0,01)$ & $0,52(0,08)$ \\
\hline Fibra modificada - cloreto de estearoil 1 mL/g & $54,9(7,02)$ & $0,167(0,04)$ & $0,48(0,10)$ \\
\hline
\end{tabular}

Tabela 3 - Propriedades mecânicas dos compósitos obtidas nos ensaios de flexão aos 28 dias de idade Resultados médios com desvios padrão entre parênteses

\begin{tabular}{c|c|c}
\hline & $\begin{array}{c}\text { Compósito com } \\
\text { fibra sem } \\
\text { tratamento }\end{array}$ & $\begin{array}{c}\text { Compósito com fibra modificada - } \\
\text { cloreto de octanoíla } \mathbf{0 , 7 5} \mathbf{~ m L / g}\end{array}$ \\
\hline Módulo de ruptura (MPa) & $3,86(0,14)$ & $4,64(0,87)$ \\
Módulo de elasticidade $(\mathbf{G P a})$ & $12,26(1,14)$ & $15,10(1,27)$ \\
Tenacidade $\left(\mathbf{k J} / \mathbf{m}^{2}\right)$ & $0,69(0,24)$ & $0,72(0,15)$ \\
\hline
\end{tabular}

Figura 15 - Ensaio de flexão - Resultado típico do compósito reforçado com fibra sem tratamento

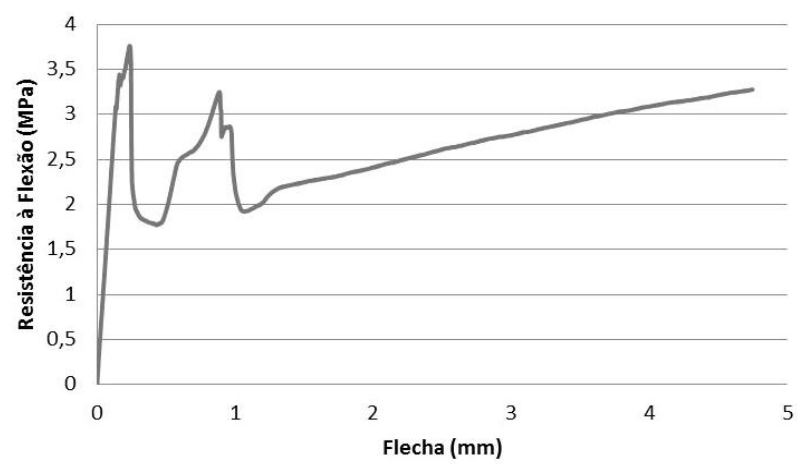

Figura 16 - Ensaio de flexão - Resultado típico do compósito reforçado com fibra modificada com cloreto de octanoíla a $0,75 \mathrm{~mL} / \mathrm{g}$

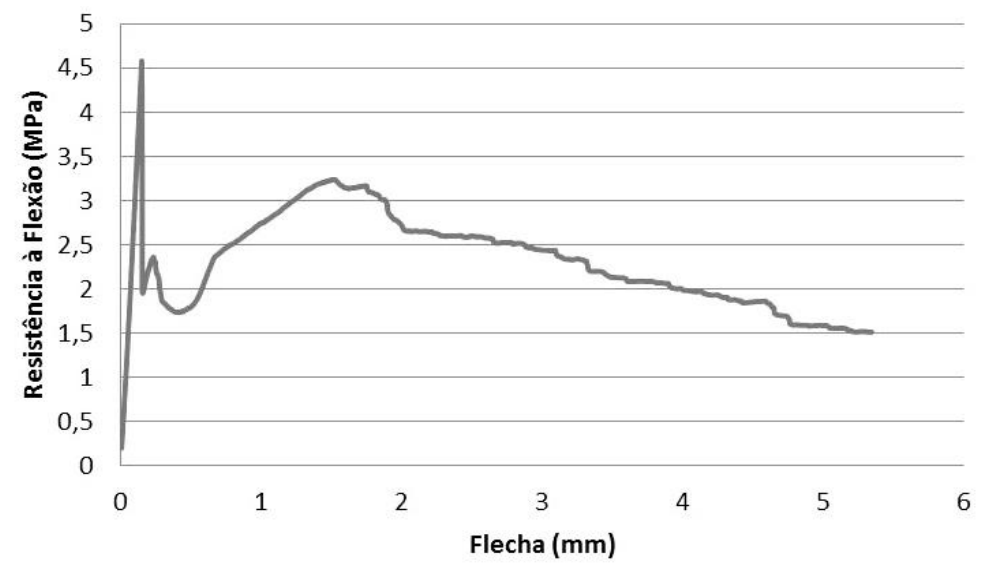

280 Souza, J. D. G. T. de; Motta, L. A. de C.; Pasquini, D.; Vieira, J. G. Pires, C. 


\section{Referências}

AGOPYAN, V. et al. Developments on Vegetable Fibre-Cement Based Materials in São Paulo, Brazil: an overview. Cement and Concrete Composites, v. 27, p. 527-536, 2005.

ANNUNCIADO, T. R. Estudo da Chorisia speciosa e Outras Fibras Vegetais Como Sorventes Para o Setor de Petróleo. Curitiba, 2005. 106 f. Dissertação (Mestrado em Engenharia e Ciência dos Materiais) - Escola de Engenharia, Universidade Federal do Paraná, Curitiba, 2005.

BISOGNIN, D. A. Origin and Evolution of Cultivated Cucurbits. Ciência Rural, Santa Maria, v. 32, n. 5, p. 715-723, 2002.

CARVALHO, J. D. V. Dossiê técnico: cultivo de bucha vegetal. Brasília: Centro de Apoio ao Desenvolvimento Tecnológico da Universidade de Brasília, 2007.

D’ALMEIDA, A. L. F. S. et al. Acetilação de Fibra de Bucha (Luffa cylindrica). Polímeros: Ciência e Tecnologia, v. 15, n. 1, p. 59-62, 2005.

FARUK, A. K. et al. Biocomposites Reinforced with Natural Fibers: 2000-2010. Progress in Polymer Science, v. 37, p. 1552-1596, 2012.

FERREIRA, S. R. et al. Influência de Ciclos de Molhagem-Secagem em Fibras de Sisal Sobre a Aderência Com Matrizes de Cimento PORTLAND. Revista Matéria, v. 17, n. 2, p. 1024-1034, 2012.

FIORE, V. et al. A New Eco-Friendly Chemical Treatment of Natural Fibres: effect of sodium bicarbonate on properties of sisal fibre and its epoxy composites. Composites Part B:

Engineering, v. 85, p. 150-160, 2015.

FREIRE, C. S. R. et al. Controlled Heterogeneous Modification of Cellulose Fibers With Fatty Acids: effect of reaction conditions on the extent of esterification and fiber properties. Journal of Applied Polymer Science, v. 100, p. 1093-1102, 2006a.

FREIRE, C. S. R. et al. Surface Characterization by XPS, Contact Angle Measurements and ToFSIMS of Cellulose Fibers Partially Esterified With Fatty Acids. Journal of Colloid and Interface Science, v. 301, p. 205-209, 2006 b.

GATENHOLM, P.; FELIX, J. Wood

Fiber/Polymer Composites: fundamental concepts, process and material options. Madison: Forest Products Society, 1993.
HOSSEINPOURPIA, R. et al. Production of Waste Bio-Fiber Cement-Based Composites Reinforced With Nano-SiO $\mathrm{S}_{2}$ Particles as a Substitute For Asbestos Cement Composites. Construction and Building Materials, v. 31, p. 105-111, 2012.

INSTITUTO BRASILEIRO DE GEOGRAFIA E ESTATÍSTICA. [Home]. Disponível em: http://www.ibge.gov.br/home/. Acesso em: 16 abr. 2016.

MARINELLI, A. L. et al. Desenvolvimento de Compósitos Poliméricos Com Fibras Vegetais Naturais da Biodiversidade: uma contribuição para a sustentabilidade amazônica. Polímeros: Ciência e Tecnologia, v. 18, n. 2, p. 92-99, 2008.

MOTTA, L. A. C. Melhoria do Desempenho de Fibras de Coco e Sisal Para Reforço de Matrizes Cimentícias Através de Tratamento Termomecânico e Impregnação de Resinas. São Paulo, 2005. 133 f. Tese (Doutorado em Engenharia Civil) - Escola Politécnica, Universidade de São Paulo, São Paulo, 2006.

MOTTA, L. A. C.; JOHN V. M.; AGOPYAN V. Thermo-Mechanical Treatment to Improve Properties of Sisal Fibres for Composites.

Materials Science Forum, v. 636-637, p. 253259, 2010.

PASQUINI, D. et al. Surface Esterification of Cellulose Fibers: characterization by DRIFT and contact angle measurements. Journal of Colloid and Interface Science, v. 295, n. 1, p. 79-83, 2006.

PASQUINI, D. et al. Surface Esterification of Cellulose Fibres: processing and characterisation of low-density polyethylene/cellulose fibres composites. Composites Science and Technology, v. 68, p. 193-201, 2008.

SAVASTANO JÚNIOR, H. Materiais à Base de Cimento Reforçados Com Fibra Vegetal: reciclagem de resíduos para a construção de baixo custo. São Paulo, 2000. 152 f. Tese (LivreDocência) - Escola Politécnica, Universidade de São Paulo, São Paulo, 2000.

SHEN, J. et al. Water-Responsive Rapid Recovery of Natural Cellular Material. Journal of the Mechanical Behaviour Biomedical Materials, v. 34, p. 283-293, 2014.

SILVA, F. A. Durabilidade e Propriedades Mecânicas de Compósitos Cimentícios Reforçados por Fibras de Sisal. Rio de Janeiro, 2009. 243 f. Tese (Doutorado em Engenharia Civil) - Escola de Engenharia, Universidade Federal do Rio de Janeiro, Rio de Janeiro, 2009. 
TANOBE, V. O. A. Caracterização de Fibras de Esponjas de Luffa cylindrica Para Utilização em Compósitos Com Matriz Polimérica. Curitiba, 2003. 161 f. Dissertação (Mestrado em Engenharia de Materiais e Processos) - Universidade Federal do Paraná, Curitiba, 2003.

TANOBE, V. O. A. et al. Influência dos Tratamentos Químicos nas Propriedades FísicoQuímicas das Esponjas de Luffa cylindrica. In: CONGRESSO BRASILEIRO DE ENGENHARIA E CIÊNCIA DOS MATERIAIS, 15., Natal, 2002. Anais... Natal, 2002.

TECHNICAL ASSOCIATION OF PULP AND PAPER INDUSTRY. TAPPI T222 om-98: acidinsoluble lignin in wood and pulp. Atlanta, 1999.

TECHNICAL ASSOCIATION OF PULP AND PAPER INDUSTRY. TAPPI T235 cm-00: alkali solubility of pulp at $25^{\circ} \mathrm{C}$. Atlanta, 2000.

TOLÊDO FILHO, R. D. et al. Development of Vegetable Fibre-Mortar Composites of Improved Durability. Cement and Concrete Composites, v. 25, p. 185-196, 2003.
TOLÊDO FILHO, R. D. et al. Durability of Alkali-Sensitive Sisal and Coconut Fibres in Cement Mortar Composites. Cement and Concrete Composites, v. 22, p. 127-143, 2000.

TONOLI, G. H. D. et al. Processing and Dimensional Changes of Cement Based Composites Reinforced With Surface-Treated Cellulose Fibers. Cement and Concrete Composites, v. 37, p. 68-75, 2013.

WEI, L.; MEYER, C. Degradation Mechanisms of Natural Fiber in the Matrix of Cement Composites. Cement and Concrete Research, v. 73, p. 1-16, 2015.

\section{Agradecimentos}

Os autores agradecem à Coordenação de Aperfeiçoamento de Pessoal de Nível Superior (Capes) por disponibilizar o portal de periódicos www.periodicos.capes.gov.br, e ao Programa de Apoio à Pós-Graduação (PROAP). José Diego G. T. de Souza agradece à Fundação de Amparo à Pesquisa do Estado de Minas Gerais (Fapemig) pela bolsa de iniciação científica (FAPEMIG 2013-ENG007), e Júlia G. Vieira agradece à Capes pela bolsa de pós-doutorado (PNPD/CAPES 20131346 - 32006012019 P5).

\footnotetext{
José Diego Gasques Tolentino de Souza

Faculdade de Engenharia Civil | Universidade Federal de Uberlândia | Rua São Pedro, 2265, Centro | Mirassol - SP - Brasil | CEP 15130000 | Tel: (17) 99673-9792 | E-mail: jdgasques@gmail.com

\section{Leila Aparecida de Castro Motta}

Faculdade de Engenharia Civil | Universidade Federal de Uberlândia | Av. João Naves de Ávila, 2121, Campus Santa Mônica, Bloco 1Y, sala 1Y 244, Santa Mônica | Caixa Postal 593 | Uberlândia - MG - Brasil | CEP 38400-902 | Tel.: (34) 3239-4173 | E-mail: lacastro@ufu.br

\section{Daniel Pasquini}

Instituto de Química | Universidade Federal de Uberlândia | Av. João Naves de Ávila, 2121, Campus Santa Mônica, Bloco 1D, Santa Mônica | Uberlândia - MG - Brasil | CEP 38400-902 | Tel.: (34) 3239-4143 | E-mail: daniel.pasquini@ufu.br

Júlia Graciele Vieira

Faculdade de Ciências Integradas do Pontal | Universidade Federal de Uberlândia | Rua Vinte, 1600, Tupã | Ituiutaba - MG - Brasil | CEP 38304-402 | Tel.: (034) 3271-5236 | E-mail: juliavieira.quimica@gmail.com
}

\title{
Cristiane Pires
}

Faculdade de Engenharia Civil | Universidade Federal de Uberlândia | Av. João Naves de Ávila, 2121, Campus Santa Mônica, Bloco 1Y, | sala 1Y 138, Santa Mônica | Uberlândia - MG - Brasil | CEP 38400-902 | Tel.: (34) 3239-4266 | E-mail: cristianepires@ufu.br

\author{
Revista Ambiente Construído \\ Associação Nacional de Tecnologia do Ambiente Construído \\ Av. Osvaldo Aranha, $99-3^{\circ}$ andar, Centro \\ Porto Alegre - RS - Brasil \\ CEP 90035-190 \\ Telefone: +55 (51) 3308-4084 \\ Fax: +55 (51) 3308-4054 \\ www.seer.ufrgs.br/ambienteconstruido \\ E-mail: ambienteconstruido@ufrgs.br
}

282 Souza, J. D. G. T. de; Motta, L. A. de C.; Pasquini, D.; Vieira, J. G. Pires, C. 